\title{
Neutron beta decay and the current determination of $V_{u d}$.
}

\author{
A. García, J.L. García-Luna ${ }^{1}$ and G. López Castro ${ }^{2}$ \\ Departamento Física, Centro de Investigación y de Estudios Avanzados del IPN, \\ Apdo. Postal 14-740, 07000 México, D.F., México
}

\begin{abstract}
Measurements of neutron beta decay observables required to determine $\left|V_{u d}\right|$ are reaching the $0.1 \%$ accuracy. In this paper we review the calculation of the decay rate of this process, discuss its relevant uncertainties, and obtain an expression that is precise at the $10^{-4}$ level. Our analysis clearly shows the necessity of more precise measurements of $\lambda$, the ratio of axial/vector couplings. The current situation in neutron beta decay is that one cannot yet quote a single consistent value for $\left|V_{u d}\right|$ from it. We also discuss the region of parameter space in the $\left|V_{u d}\right|-\lambda$ plane where new physics effects should lie, if they contribute to neutron beta decay.
\end{abstract}

1. Introduction. Neutron beta decay can provide in the future the most precise determination of the Cabibbo-Kobayashi-Maskawa (CKM) matrix element $\left|V_{u d}\right|[1]$. The experimental inputs required for this purpose are the neutron lifetime $\tau_{n}$ and the ratio of axial-vector to vector couplings, $\lambda \equiv$ $g_{1}(0) / f_{1}(0)$. An update of the precise computation of the neutron decay rate is important for (at least) the following reasons: (a) the measurement of $\tau_{n}$ and $\lambda$ have reached the $0.1 \%$ accuracy and further improvements are expected in the future (see for example [2]) and (b) independent and precise determination of $V_{u d}$ is compelling to test the unitarity condition $\sum_{i=d, s, b}\left|V_{u i}\right|^{2}=1$ of the CKM matrix. This requires from theory the computation of the decay rate at the $10^{-4}$ level in accuracy, and will allow a determination of $\left|V_{u d}\right|$ with a precision $0.1 \%$ which becomes competitive with the determination coming from superallowed Fermi transitions (SFT) among $J^{P}=0^{+}$nuclei [3], namely,

$$
\left|V_{u d}\right|=0.9740 \pm 0.0010 \text {. }
$$

The purpose of the present paper is to review the computation of the neutron

1 E-mail: jlgarcia@fis.cinvestav.mx

2 E-mail: glopez@fis.cinvestav.mx

Preprint submitted to Elsevier Preprint

20 November 2018 
beta decay rate and discuss its relevant uncertainties at the $10^{-4}$ level. At this level it becomes necessary to discuss the relevance of the induced hadronic form factors and even radiative corrections up to $\alpha^{2}$ contributions $[4,5]$.

There are two reasons why $\left|V_{u d}\right|$ can be obtained from SFT with very small error bars. One is of statistical nature, because Eq.(1) arises from the weighted average of 9 measured transitions [6]. The other one is an advantage of SFT over neutron beta decay; they involve at tree-level only the vector current. The present uncertainty in $\left|V_{u d}\right|$ from SFT has two dominant (theoretical) sources: the nuclear-dependent (mismatch) isospin breaking corrections and the piece of photonic corrections induced by the axial-vector current $[7,8]$. The advantage of neutron beta decay is that it is free of a nuclear environment, but its (theoretical) disadvantage is that it involves at tree-level the axial-vector current, too. Away from a nuclear environment, the isospin-breaking corrections are more reliable to compute. Such corrections clearly affect both leading form factors. However, the isospin breaking corrections enter into $f_{1}(0)$ only at second order in the breaking by virtue of the Ademollo-Gatto theorem [9]. This fact makes them very small and they contribute only at the $10^{-5}$ level [10]. On the other hand, these corrections are more important in $g_{1}(0)$ and, also, they are more difficult to compute reliably. This complication is avoided using the experimental value of $g_{1}(0)$, through the measurement of the ratio $\lambda$. The main source for such measurements is the electron-neutron-spin asymmetry coefficient. It is because the experimental uncertainty in $\lambda$ can be much improved over its theoretical uncertainty that the neutron beta decay determination of $\left|V_{u d}\right|$ may become its best determination.

For sakeness of clarity we will present the different ingredients for the determination of the decay rate in the following order. In section 2 we provide the expression for the decay rate of the neutron at tree-level by including small effects of non-leading form factors (other than $f_{1}(0)$ and $g_{1}(0)$ ). In section 3 we address the issue of radiative corrections. We will discuss the uncertainties associated with factorization of different orders in $\alpha$ as commonly presented in the literature. In section 4 we summarize the current information of experimental data on $\tau_{n}$ and $\lambda$ including the most recent results. In particular, we will discuss the discrepancies in different measurements of $\lambda$, and we will provide a comparison of the different determinations of $\left|V_{u d}\right|$ as obtained from different sources. Section 5 contains our conclusions.

2. Decay rate at the tree-level. The tree-level amplitude (meaning that radiative corrections are ignored) for the decay $n \rightarrow p e^{-} \bar{\nu}_{e}$ can be parametrized in terms of six Lorentz-invariant form factors. The most general form of this amplitude is given by (we follow the conventions of Ref. [5]):

$$
M^{0}=\frac{G_{0}}{\sqrt{2}} V_{u d} \bar{u}\left(p^{\prime}\right)\left\{f_{1}\left(q^{2}\right) \gamma_{\mu}+\frac{f_{2}\left(q^{2}\right)}{m_{n}} \sigma_{\mu \nu} q^{\nu}+\frac{f_{3}\left(q^{2}\right)}{m_{n}} q_{\mu}\right.
$$




$$
\left.+\left[g_{1}\left(q^{2}\right) \gamma_{\mu}+\frac{g_{2}\left(q^{2}\right)}{m_{n}} \sigma_{\mu \nu} q^{\nu}+\frac{g_{3}\left(q^{2}\right)}{m_{n}} q_{\mu}\right] \gamma_{5}\right\} u(p) l^{\mu}
$$

where $G_{0}$ is the effective weak coupling of the relevant four-fermion hamiltonian at the tree-level and $l_{\mu}$ is the leptonic V-A current. $f_{i}, g_{i}$ denote dimensionless $q^{2}=\left(p-p^{\prime}\right)^{2}$ dependent form factors. Since the momentum transfer is very small $\left(q_{\max }^{2} / m_{n}^{2} \approx 1.9 \times 10^{-6}\right)$, we can already anticipate that the contributions of the form factors $f_{2}, f_{3}, g_{2}$ and $g_{3}$ to the decay rate are very suppressed. By the same reason, the $q^{2}$ dependence of form factors can be safely neglected.

By including recoil effects (in the phase space factor, too), the rate of neutron beta decay can be written as [5]:

$$
\begin{aligned}
R^{0}= & \frac{G_{0}^{2} m_{e}^{5}}{2 \pi^{3}}\left|V_{u d}\right|^{2}\left[1.63387 f_{1}^{2}+2.3775 \cdot 10^{-6} f_{1} f_{2}+1.1625 \cdot 10^{-3} f_{1} f_{3}+\right. \\
& 1.5836 \cdot 10^{-6} f_{2}^{2}+3.1835 \cdot 10^{-6} f_{1} \lambda_{f_{1}}+4.90159 g_{1}^{2}-6.2017 \cdot 10^{-6} g_{2}^{2} \\
& \left.-3.1697 \cdot 10^{-7} g_{1} g_{3}-1.0161 \cdot 10^{-2} g_{1} g_{2}+1.1453 \cdot 10^{-5} g_{1} \lambda_{g_{1}}\right]
\end{aligned}
$$

where $m_{e}$ is the mass of the electron and $f_{i}, g_{i}$ are form factors evaluated at zero momentum transfer. The coefficients $\lambda_{f_{i}}, \lambda_{g_{i}}$ denote the corrections due to $q^{2}$ dependence of $f_{i}$ and $g_{i}$. We do not display the contributions of $f_{3}^{2}, g_{3}^{2}$, $\lambda_{f_{2}}, \lambda_{g_{2}}$ and $\lambda_{g_{3}}$ since their coefficients are at the $10^{-8}$ level, i.e., well below the current experimental precision. In Eq.(3) we improved the precision of the coefficients of the leading form factors by one more decimal place, with respect to Ref. [5].

In order to get a simpler expression that incorporates the terms in Eq. (3) relevant at order $10^{-4}$ let us discuss the size of the different contributions. As is well known, in the limit of exact isospin symmetry the conserved current hypothesis (CVC) allows one to obtain $f_{1}^{C V C}=1$ and $f_{2}^{C V C}=1.8529$ [5]. Also, exact G-parity, that is expected to hold in the isospin symmetry limit, predicts the absence of second class form factors: $f_{3}=0$ and $g_{2}=0$. The contribution of the axial form factor $g_{3}$ to the decay rate is expected to be below the $10^{-4}$ level, even if, according to the PCAC hypothesis, $g_{3}=\left(2 m_{n}^{2} / m_{\pi}^{2}\right) g_{1} \approx 114$.

When isospin symmetry gets broken (due to the mass difference of $u$ and $d$ quarks), the above predictions change. For example, $f_{1}(0)$ will deviate slightly from unity while $g_{2}$ and $f_{3}$ become different from zero. An estimate of these effects indicates that $1-f_{1} \approx 5 \times 10^{-5}[10], g_{2} \approx 6 \times 10^{-3}$ and $f_{3} \sim 10^{-3}$ [11]. Therefore, the expression for the decay rate at the lowest order that is precise at the $10^{-4}$ level gets the simple form:

$$
R^{0}=\frac{G_{0}^{2} m_{e}^{5}}{2 \pi^{3}}\left|V_{u d}\right|^{2}(1.6335)\left(1+3 \lambda^{2}\right)
$$

3. Radiative corrections. An expression for the decay rate that is 
precise at this level requires the inclusion of radiative corrections up to order $\alpha^{2}$. The formal perturbative expansion in powers of $\alpha$ for the decay rate can be expressed as follows:

$$
R=R^{0}+R(\alpha)+R\left(\alpha^{2}\right)+\cdots
$$

where $R^{0}$ is the rate at tree-level discussed in the previous section and $R\left(\alpha^{n}\right)$ denote its corrections of order $\alpha^{n}$.

At any given order in $\alpha$, the radiative corrections can be split into two terms: $R\left(\alpha^{n}\right)=R_{M I}\left(\alpha^{n}\right)+R_{M D}\left(\alpha^{n}\right)$, the model-independent (MI) and the model-dependent (MD) pieces, following their independence or not of the details of weak and strong interactions.

The MI radiative corrections, also called inner corrections, arise from longdistance QED (virtual and bremsstrahlung) corrections up to an energy scale of $O(1 \mathrm{GeV})$. The MD radiative corrections, also called outer corrections, involve essentially the short-distance electroweak corrections.

In the following sections we will discuss in detail the leading orders of radiative corrections that have been computed in the literature.

3.1 Corrections of order $\alpha$. The corrections of order $\alpha$ have been computed long ago $[12,13]$. The model-independent piece of $O(\alpha)$ was given as [12]

$$
R_{M I}(\alpha)=\frac{G_{F}^{2} m_{e}^{5}}{2 \pi^{3}}\left|V_{u d}\right|^{2}\left(1+3 \lambda^{2}\right) \frac{1}{m_{e}^{5}} \int\left(f_{\alpha}+\frac{\alpha}{2 \pi} g(E)\right) d S
$$

while the model-dependent contribution as [13]

$$
\begin{aligned}
R_{M D}(\alpha)= & \frac{G_{F}^{2} m_{e}^{5}}{2 \pi^{3}}\left|V_{u d}\right|^{2}\left(1+3 \lambda^{2}\right) \frac{1}{m_{e}^{5}} \int \frac{\alpha}{2 \pi}\left(3 \ln \frac{m_{W}}{m_{p}}+\ln \frac{m_{W}}{m_{A}}+\right. \\
& \left.2 C-4 \ln \frac{m_{W}}{m_{Z}}+A_{g}\right) d S .
\end{aligned}
$$

In the above equations $E$ denotes the energy of the electron, $l=|\vec{l}|$ its threemomentum and $d S \equiv E l\left(E_{m}-E\right)^{2} d E$. The function $f_{\alpha}$ denotes the coulombic correction of order $\alpha$,

$$
f_{\alpha}=\frac{\alpha}{\pi} \frac{\pi^{2} E}{l}
$$

and the expression for $g(E)$ can be found in Ref. [12]. The origin of the different terms in Eq. (7) can be found elsewhere [13,7]. Numerically, the MI radiative corrections at this order are

$$
\frac{1}{m_{e}^{5}} \int f_{\alpha} d S=0.0546
$$




$$
\frac{1}{m_{e}^{5}} \int \frac{\alpha}{2 \pi} g(E) d S=0.0246
$$

As we can observe, the dominant piece of MI radiative corrections of order $\alpha$ comes from the coulombic term. We will leave the numerical evaluation of the MD corrections to the following subsection.

Before we close this subsection let us emphasize that the remaining effects of radiative corrections that have a universal nature have been absorbed in the redefinition of the effective weak coupling constant with the result $G_{0} \rightarrow G_{F}$, where $G_{F}=1.16639(2) \times 10^{-5} \mathrm{GeV}^{-2}$ can be extracted from muon decay [3]. 3.2 Corrections of order $\alpha^{2}$. In this subsection we consider the MI radiative corrections of $O\left(\alpha^{2}\right)$ [14]. The MD corrections of this and higher orders will be discussed in subsection 3.3. In a similar way to the previous subsection, we can write the MI corrections as [4]:

$$
R_{M I}\left(\alpha^{2}\right)=\frac{G_{F}^{2} m_{e}^{5}}{2 \pi^{3}}\left|V_{u d}\right|^{2}\left(1+3 \lambda^{2}\right) \frac{1}{m_{e}^{5}} \int\left(f_{\alpha^{2}}+\delta_{\alpha^{2}}\right) d S
$$

where again, we have separated explicitly the coulombic correction $f_{\alpha^{2}}$ from the remaining corrections $\delta_{\alpha^{2}}$ at this order.

Numerically, we have:

$$
\begin{aligned}
\frac{1}{m_{e}^{5}} \int f_{\alpha^{2}} d S & =0.0014 \\
\frac{1}{m_{e}^{5}} \int \delta_{\alpha^{2}} d S & =0.0007 .
\end{aligned}
$$

As in the case of order $\alpha$, we observe the dominance of the coulombic piece in the MI radiative corrections of order $\alpha^{2}$.

3.3 Corrections of order higher than $\alpha^{2}$. The leading pieces of MI radiative corrections of order $\alpha^{3}$ have been computed in Ref. [14]. Following the pattern already observed, we can expect that the MI corrections will be dominated also by the coulombic term. Indeed, coulombic corrections can be included to all orders (via the Fermi function $F(Z, E)[15]$ ) by solving the non-relativistic Schrödinger equation for the electron in the Coulomb field of the proton. However, it it clear that for the purposes of our analysis, we have to keep only the MI corrections up to $O\left(\alpha^{2}\right)$.

Now, we will focus on the MD radiative corrections. Following the procedure discussed in $[13,7]$, let us rewrite the MD corrections of $O(\alpha)$ in the form

$$
R_{M D}(\alpha)=\frac{G_{F}^{2} m_{e}^{5}}{2 \pi^{3}}\left|V_{u d}\right|^{2}\left(1+3 \lambda^{2}\right) \frac{1}{m_{e}^{5}} \int \frac{\alpha}{2 \pi}\left(4 \ln \frac{m_{Z}}{m_{p}}+\ln \frac{m_{p}}{m_{A}}\right.
$$




$$
\left.+2 C+A_{g}\right) d S
$$

The term $\ln \left(m_{Z} / m_{p}\right)$ is by far the dominant piece of this correction. As in the case of coulombic corrections, one may wonder about the relative size of higher order MD corrections having logarithmic terms. Ref. [7] has pointed out that the effects of higher order can be approximated by resummation of the leading-logarithmic corrections of $O\left(\alpha^{n} \ln ^{2}\left(m_{Z} / m_{p}\right)\right), n=1,2, \cdots$ via the renormalization group equation (RGE). The net effect of this resummation is to replace Eq. (14) by [7]:

$$
\begin{gathered}
R_{M D}^{\prime}=\frac{G_{F}^{2} m_{e}^{5}}{2 \pi^{3}}\left|V_{u d}\right|^{2}\left(1+3 \lambda^{2}\right) \frac{1}{m_{e}^{5}} \int\left[\frac{\alpha}{2 \pi}\left(\ln \frac{m_{p}}{m_{A}}+2 C+A_{g}\right)\right. \\
\left.+S\left(m_{p}, m_{Z}\right)-S\left(m_{p}, m_{p}\right)\right] d S,
\end{gathered}
$$

where $R_{M D}^{\prime}$ denotes the MD radiative corrections that take into account dominant logarithmic corrections at higher orders.

According to ref. [7], $S\left(m_{p}, m_{Z}\right)$ can be obtained by solving an specific RGE subject to the boundary condition condition $S\left(m_{p}, m_{p}\right)=1$. The numerical result for this correction is:

$$
S\left(m_{p}, m_{Z}\right)=1.0220
$$

To obtain this numerical value, which differs slightly from Ref. [7], we have used the fact that the top quark is heavier than the $Z$ boson and should not be included in the thresholds when solving the RGE.

3.4 Final remarks on radiative corrections. If we collect the results presented in the previous sections, we can write the radiative corrections to the neutron decay rate as

$$
\begin{aligned}
& \sum_{n} R\left(\alpha^{n}\right)=\frac{G_{F}^{2} m_{e}^{5}}{2 \pi^{3}}\left|V_{u d}\right|^{2}\left(1+3 \lambda^{2}\right)\left\{\frac { 1 } { m _ { e } ^ { 5 } } \int \left[f_{\alpha}+\frac{\alpha\left(m_{p}\right)}{2 \pi} g(E)+f_{\alpha^{2}}\right.\right. \\
& \left.\left.+\delta_{\alpha^{2}}+\frac{\alpha}{2 \pi}\left[\ln \left(\frac{m_{p}}{m_{A}}\right)+2 C\right]+\frac{\alpha\left(m_{p}\right)}{2 \pi} A_{g}+S\left(m_{p}, m_{Z}\right)-S\left(m_{p} m_{p}\right)\right] d S\right\} .
\end{aligned}
$$

In some terms of the above equations we have replaced $\alpha^{-1}(0)=137.036 \rightarrow$ $\alpha^{-1}\left(m_{p}\right)=133.93$. According to Ref. [7] $m_{p}$ is the relevant scale for the electromagnetic coupling in the renormalization scheme adopted in these calculations.

Let us stress that Eq. (17) has the correct additive structure in terms of the expansion parameter $\alpha$. This observation is very important in view of several factorization approximations currently found in the literature [4,8] which cannot be justified when the decay rate is computed at order $10^{-4}$. For example, 
it is customary to take the coulombic corrections to all orders through the Fermi function as a global factor in the integrand of Eq. (17) [4]. This would lead to the following expression for the decay rate

$$
\begin{gathered}
R=\frac{G_{F}^{2} m_{e}^{5}}{2 \pi^{3}}\left|V_{u d}\right|^{2}\left(1+3 \lambda^{2}\right)\left\{\frac { 1 } { m _ { e } ^ { 5 } } \int F ( Z , E ) \left[1+\frac{\alpha\left(m_{p}\right)}{2 \pi} g(E)+\delta_{\alpha^{2}}\right.\right. \\
\left.\left.+\frac{\alpha}{2 \pi}\left[\ln \left(\frac{m_{p}}{m_{A}}\right)+2 C\right]+\frac{\alpha\left(m_{p}\right)}{2 \pi} A_{g}+S\left(m_{p}, m_{Z}\right)-S\left(m_{p}, m_{p}\right)\right] d S\right\}
\end{gathered}
$$

Clearly, this factorization introduces spurious terms of order $\alpha^{2}$, through the product of the term of order $\alpha$ in the Fermi function with other contributions of $O(\alpha)$ in the remaining corrections. A similar argument can be applied to the factorization of outer and inner radiative corrections (see ref. [7] and Eq. (7) in Ref. [8]). Therefore, in the computation of the decay rate at the $10^{-4}$ level (or to a higher accuracy) radiative corrections must be taken in an additive form as shown in Eq.(5) in order to avoid spurious corrections of order $\alpha^{2}$ (and higher orders).

After these remarks, we proceed to obtain a numerical expression for the decay rate involving radiative corrections. The numerical values of some of these corrections have been provided in Eqs. (9,10, 12, 13 and 16). Currently, the largest uncertainty in radiative corrections comes from the terms which originate in the axial-induced photonic corrections,

$$
\Delta \equiv \frac{\alpha}{2 \pi}\left[\ln \left(\frac{m_{p}}{m_{A}}\right)+2 C\right]
$$

The non-asymptotic piece $C$ has been re-evaluated in ref. [8] with the result $C=0.881 \pm 0.030$. The mass parameter $m_{A}$ is taken as a low energy cutoff applied to the evaluation of the axial part of the asymptotic $\gamma W$ box correction. Marciano and Sirlin [7] have suggested that, given the ambiguities in the choice of this cutoff, it is more appropriate to take the range $400 \mathrm{MeV} \leq m_{A} \leq 1600 \mathrm{MeV}$ for this axial mass. This wide range, is at present the major source of uncertainty in radiative corrections to neutron beta decay, since the term given in Eq. (19) becomes

$$
\Delta=0.0022 \pm 0.0008 \text {. }
$$

If we insert the different values of radiative corrections discussed above, we obtain an expression of the decay rate that is accurate at the $10^{-4}$ level:

$$
R=0.1897\left|V_{u d}\right|^{2}\left(1+3 \lambda^{2}\right)(1+0.0739 \pm 0.0008) \times 10^{-3} \mathrm{~s}^{-1},
$$

where the quoted error comes from the uncertainty in the axial cutoff param- 
eter $[7,8]$.

Notice that if we had included radiative corrections with the Fermi function $F(Z, E)$ and $\mathrm{MD}$ corrections in a factorized form, we would have obtained,

$$
R=0.1897\left|V_{u d}\right|^{2}\left(1+3 \lambda^{2}\right)(1+0.0758 \pm 0.0008) \times 10^{-3} \mathrm{~s}^{-1},
$$

to be compared to Eq. (20).

As it was previously mentioned, the factorization of some pieces of radiative corrections affects the expression for the decay rate at the order $10^{-4}$. Therefore, in the following we will use our Eq. (20) to quote a determination of $\left|V_{u d}\right|$.

4. Determination of $\left|V_{u d}\right|$. Comparison with other sources. According to Eq. (20), two experimental inputs are required to determine $\left|V_{u d}\right|$ from neutron beta decay: the neutron lifetime $\tau_{n} \equiv R^{-1}$ and $\lambda$. We first summarize here the current situation regarding their measurements and afterwards we discuss $\left|V_{u d}\right|$.

4.1 Neutron lifetime. The world average reported by the Particle Data Group in the 1998 edition is [3]

$$
\tau_{n}^{P D G 98}=\frac{1}{R^{e x p}}=(886.7 \pm 1.9) \mathrm{s}
$$

This value is obtained [3] from a weighted average of ten independent measurements and the quoted error has been inflated by a scale factor $\mathrm{S}=1.2$. A more recent and more precise measurement has been reported in the literature $[6,20,22]: \tau_{n}=(885.4 \pm 1.0) \mathrm{s}$, which is consistent with Eq. (22). Therefore, from its weighted average with Eq. (22), we obtain

$$
\tau_{n}^{\text {new }}=(885.9 \pm 0.9) \mathrm{s} .
$$

The set of measurements of $\tau_{n}$ is consistent as reflected by a low value of the scale factor $(\mathrm{S}=1.07)$. As it was mentioned in the introduction, the measurement of $\tau_{n}$ has reached the impressive accuracy of $0.1 \%$.

4.2 Ratio $\lambda=g_{1}(0) / f_{1}(0)$. The consistency of measurements of $\lambda$ is not clear at present. So far, measurements of the electron spin asymmetry have provided the most precise values reported for $\lambda$. By combining the four most precise measurements (typically those with an accuracy higher than $0.4 \%$ ) one gets the weighted average [3]

$$
\lambda^{P D G 98}=1.2670 \pm 0.0035
$$

where the error has been increased by an scale factor of $S=1.9$. Therefore, the choice of this set of measurements would indicate a precision of $0.3 \%$ in 
$\lambda$. Other less precise measurements may be excluded because they have negligible statistical weight at the $10^{-4}$ level.

In order to study the impact of different measurements of $\lambda$ on the determination of $\left|V_{u d}\right|$, we prefer to quote the value of this parameter as a function of different values of $\lambda$. Following Ref. [3], we will choose the four most precise measurements of $\lambda: \lambda_{i}$, where $i=L, B, Y, R$ denote, respectively, the results of references $[18,19,16,20]$. We will exclude from our analysis the result of Ref. [17], because it has been superseded by the new result in Ref. [20]. The explicit values are given in Table 1 . The updated average becomes

$$
\lambda^{P D G 99}=1.2687 \pm 0.0016
$$

where the error has not been increased by any scale factor.

Several comments about Eq. (25) are in order. First, the two most precise values of $\lambda$ used to obtain Eq. (25), namely, $\lambda_{Y}$ and $\lambda_{R}$ differ each one only by two standard deviations from Eq.(25) and by 4.7 standard deviations from each other. This reflects an inconsistency between both measurements. Second, the same inconsistency can be observed in the ideogram shown in p. 622 of ref. [3] where the set of 4 measurements used to quote Eq.(24) are grouped into two separated peaks of Gaussian distributions. This signals the lack of meaning of the single weighted average given in (24) and points out towards the necessity of new and independent measurements of $\lambda$ to elucidate the nature of the systematic errors.

4.3 Determination of $\left|V_{u d}\right|$ from neutron beta decay. In Table 1, we show the values of $\left|V_{u d}\right|$ obtained for individual measurements of $\lambda$ and some of its partial averages. Also displayed are the corresponding values of the associated scale factor $S=\sqrt{\sum_{i} \chi_{i}^{2} /(n-1)}$ when more than one value of $\lambda$ is used; the bigger the scale factor is, the larger the inconsistency within the set of chosen data. The inconsistency among the measurements of $\lambda$ can be appreciated in several ways (see Table 1): (a) the central values of $\left|V_{u d}\right|$ corresponding to $\lambda_{Y}$ and $\lambda_{R}$ differ by almost $4 \sigma,(b)$ if we use the two sets of data that appear clustered into two separated Gaussian distributions (see section 4.2), namely, $\lambda_{L Y B}$ and $\lambda_{R}$, we find that the corresponding central values of $\left|V_{u d}\right|$ differ by more than $4 \sigma$.

We observe that in the latter case $(b)$ the corresponding values of $\left|V_{u d}\right|$ violate the unitarity of the CKM matrix. Indeed, if we use $\left|V_{u s}\right|=0.2196 \pm$ 0.0023 and $\left|V_{u b}\right|=0.0033 \pm 0.0008$ [3] we obtain (for comparison we include the unitarity check for SFT)

$$
\left|V_{u d}\right|^{2}+\left|V_{u s}\right|^{2}+\left|V_{u b}\right|^{2}=\left\{\begin{array}{l}
0.9928 \pm 0.0031, \text { for } \lambda_{R} \\
0.9969 \pm 0.0022, \text { for } S F T \\
1.0066 \pm 0.0035, \text { for } \lambda_{L Y B}
\end{array}\right.
$$


It is very interesting to observe that when we combine the set of four data, namely, if we use $\lambda_{L B Y R}$, we obtain a value of $\left|V_{u d}\right|$ that is (accidentally!) consistent with unitarity $\left|V_{u d}\right|^{2}+\left|V_{u s}\right|^{2}+\left|V_{u b}\right|^{2}=0.9986 \pm 0.0025$.

Therefore, since the whole set of most precise measurements of $\lambda$ is inconsistent, we can only quote two different values of $\left|V_{u d}\right|$, namely, those corresponding to the two clearly separated Gaussian distributions shown in the ideogram of p. 622 in Ref. [3]. Explicitly (for comparison we include $\left|V_{u d}\right|$ from the unitarity of CKM matrix)

$$
\left|V_{u d}\right|=\left\{\begin{array}{l}
0.9719 \pm 0.0019 \pm 0.0004 \text { from } \lambda_{R} \\
0.9790 \pm 0.0016 \pm 0.0004 \text { from } \lambda_{L Y B} . \\
0.9756 \pm 0.0005 \text { from unitary of CKM matrix. }
\end{array}\right.
$$

The first quoted error arises mainly from the uncertainty in the measurement of $\lambda$, while the second one comes from the uncertainty in the calculation of MD radiative corrections. This result indicates that besides the interest of new measurements of $\lambda$ to solve the current discrepancy, they are also required to provide the most precise determination of $\left|V_{u d}\right|$.

One may wonder where future measurements of $\lambda$ should lie if no new physics effects are relevant in neutron beta decay. In Fig. 1. we have plotted the one standard deviation elliptical contours of $\left|V_{u d}\right|$ and $\lambda$ obtained from Eq. (20) and the two above values of $\lambda$. For comparison we also display the two horizontal bands corresponding to $\left|V_{u d}\right|$ from unitarity and SFT at one standard deviation, too.

The small ellipse in Fig. 1 within the CKM band is the one standard deviation region allowed by the unitarity $\left|V_{u d}\right|$ and Eq. (23) and it is the region where no new physics can yet appear in neutron beta decay. The current situation is that the two values of the $\left|V_{u d}\right|$ from neutron beta decay and its value from SFT do not overlap with that region. Thus, Fig. 1. provides a visualization of Eqs. (26) and (27). At any rate, neither of these discrepancies with unitarity has enough statistical significance to be currently accepted as an indication of the existence of new physics. But, the smallness of this region illustrates the potential of neutron beta decay to either detect new physics or to provide strong lower bounds on its existence.

5. Conclusions. The determination of $\left|V_{u d}\right|$ from neutron beta decay has several advantages. First, isospin breaking effects are well under control since they affect the decay rate at the level below the $10^{-4}$ (contrary to SFT where they enter at a few tenths of percent level [8]). Second, the piece of MD radiative corrections $C$ (see Eq. (19)) which is associated to the non-asymptotic axial-induced photonic corrections only receive contributions from one-body terms (contrary to SFT where the photon and the $W$ gauge boson appearing in the box diagram can be coupled to different nucleons). Third, possible residual effects of $Z$-dependent radiative corrections (see Ref. [21]) are absent 
in neutron beta decay. In addition, the non-leading form factors are irrelevant at this level of precision. The complications of determining $g_{1}(0)$ do remain. Fortunately, this form factor can be taken from experiment, because its experimental value can be made appreciably more precise than its theoretical one. Thus, the main source of theoretical uncertainties in the neutron beta decay rate are the radiative corrections. We have seen that they are well under control up to a few parts in $10^{-4}$. Prescriptions used to incorporate different contributions of these corrections become relevant. By this we mean that the (commonly used) factorization approximations of some pieces of radiative corrections introduce spurious contributions at the $10^{-4}$ level. The current prescription is the additive one.

However, new measurements of $\lambda$ are required, first, to solve the discrepancy among existing data and, second, to improve the determination of $\left|V_{u d}\right|$. Actually (see Eq. (27)), an improvement of a reduction of a factor 2 in the error bars of $\lambda$ would provide a determination of $\left|V_{u d}\right|$ that is competitive with the one coming from SFT.

The main conclusion in the above analysis is that the theoretical decay rate of neutron beta decay is well under control up to a few parts at the $10^{-4}$ level. Given the present discrepancy of experimental measurements of $\lambda$ and our limitation to combine those data in a statistically significant way, we can only quote the two favored values of $\left|V_{u d}\right|$ as given in Eq. (27). These values correspond to the two subsets of consistent data according to the ideogram shown in p. 622 of the Particle Data Group [3]. New physics effects can only be detected in neutron beta decay outside the small ellipse within the CKM matrix band in Fig. 1.

From Eq.(27) it becomes clear that, once the error bars on $\lambda$ are appreciably reduced, it will be very important to reduce the theoretical error bars. Therefore it would be very useful to invest some effort in better controlling the model dependent piece of the radiative corrections in neutron beta decay.

\section{Acknowledgments.}

The authors wish to express their gratitude to CONACyT (México). (J.L.G.L.) wishes to acknowledge a leave of absence from Universidad de Guadalajara (México).

\section{References}

[1] N. Cabibbo, Physs. Rev. Lett. 10, 351 (1963); M. Kobayashi and T. Maskawa, Prog. Theor. Phys. 49, 652 (1973).

[2] J. Deutsch, nucl-th/9901098, to appear in the WEIN 98 proceeding.

[3] C. Caso et al, Particle Data Group. E. Phys. J. C3, 1 (1998).

[4] D. Wilkinson, Nucl. Phys. A377, 474 (1982). 
[5] A. García and P. Kielanowski, Hyperon Beta Decay, Lectures Notes in Physics 222, (Springer-Verlag, 1985).

[6] I. Towner and J. C. Hardy, nucl-th/9809087.

[7] W. J. Marciano and A. Sirlin, Phys. Rev. Lett. 56, 22 (1986).

[8] I. S. Towner, Nucl. Phys. A540, 478 (1992).

[9] R.Behrends and A. Sirlin, Phys. Rev. Lett. 4, 186(1964); M. Ademollo and R. Gatto, Phys. Rev. Lett. 13, 264(1964).

[10] N. Paver and Riazzuddin, Phys. Lett. B260, 421 (1991); J. F. Donoghue and D. Wyler, Phys. Lett. B 241, 243 (1990).

[11] A. Halprin, B. W. Lee and P. Sorba, Phys. Rev. D14, 2343(1976).

[12] A. Sirlin, Phys. Rev. 164, 1767(1967).

[13] A. Sirlin, Rev. of Mod. Phys. 50, 573(1978).

[14] A. Sirlin, Phys. Rev. D37, 3423(1987); W. Jaus, Phys. Rev. D35, 3420(1987).

[15] T.A. Halpern, Phys. Rev. C1, 1928(1970).

[16] B. Yerozolimsky et al, Phys. Lett. B412, 240 (1997).

[17] H. Abele et al, Phys. Lett. B407, 212 (1997).

[18] P. Liaud et al, Nucl. Phys. A612, 53 (1997).

[19] P. Bopp et al, Phys. Rev. Lett. 56, 919 (1986).

[20] J. Reich et al, V Int. Seminar on Interaction of Neutrons with Nuclei, Dubna 1997.

[21] D. Wilkinson, Z. Phys. A 348, 129(1994).

[22] S. Arzumanov, et al, to appear in Phys. Lett. B. 


\begin{tabular}{|c|c|c|}
\hline$\lambda$ & scale factor & $\left|V_{u d}\right|$ \\
\hline$\lambda_{L}=1.2660(40)$ & - & $0.9766 \pm 0.0026$ \\
\hline$\lambda_{Y}=1.2594(38)$ & - & $0.9809 \pm 0.0025$ \\
\hline$\lambda_{B}=1.2620(50)$ & - & $0.9792 \pm 0.0032$ \\
\hline$\lambda_{R}=1.2735(21)$ & - & $0.9719 \pm 0.0015$ \\
\hline$\lambda_{L B}=1.2644(31)$ & 0.6 & $0.9777 \pm 0.0021$ \\
\hline$\lambda_{L Y}=1.2625(28)$ & 1.2 & $0.9789 \pm 0.0019$ \\
\hline$\lambda_{Y B}=1.2604(30)$ & 0.4 & $0.9802 \pm 0.0020$ \\
\hline$\lambda_{L R}=1.2719(19)$ & 1.7 & $0.9729 \pm 0.0014$ \\
\hline$\lambda_{Y R}=1.2702(18)$ & 3.2 & $0.9740 \pm 0.0013$ \\
\hline$\lambda_{B R}=1.2718(19)$ & 2.1 & $0.9730 \pm 0.0014$ \\
\hline$\lambda_{L Y B}=1.2624(24)$ & 1.6 & $0.9790 \pm 0.0017$ \\
\hline$\lambda_{L B R}=1.2707(17)$ & 1.8 & $0.9737 \pm 0.0012$ \\
\hline$\lambda_{Y B R}=1.2692(17)$ & 2.5 & $0.9746 \pm 0.0012$ \\
\hline$\lambda_{Y L R}=1.2695(17)$ & 2.4 & $0.9744 \pm 0.0012$ \\
\hline$\lambda_{L Y B R}=1.2687(16)$ & 2.1 & $0.9749 \pm 0.0012$ \\
\hline$T a b 1$ & & \\
\hline
\end{tabular}

Table 1

Values of $\left|V_{u d}\right|$ obtained from different values of $\lambda$ and $\tau=(885.9 \pm 0.9) \mathrm{s}$, via Eq. 20 . 


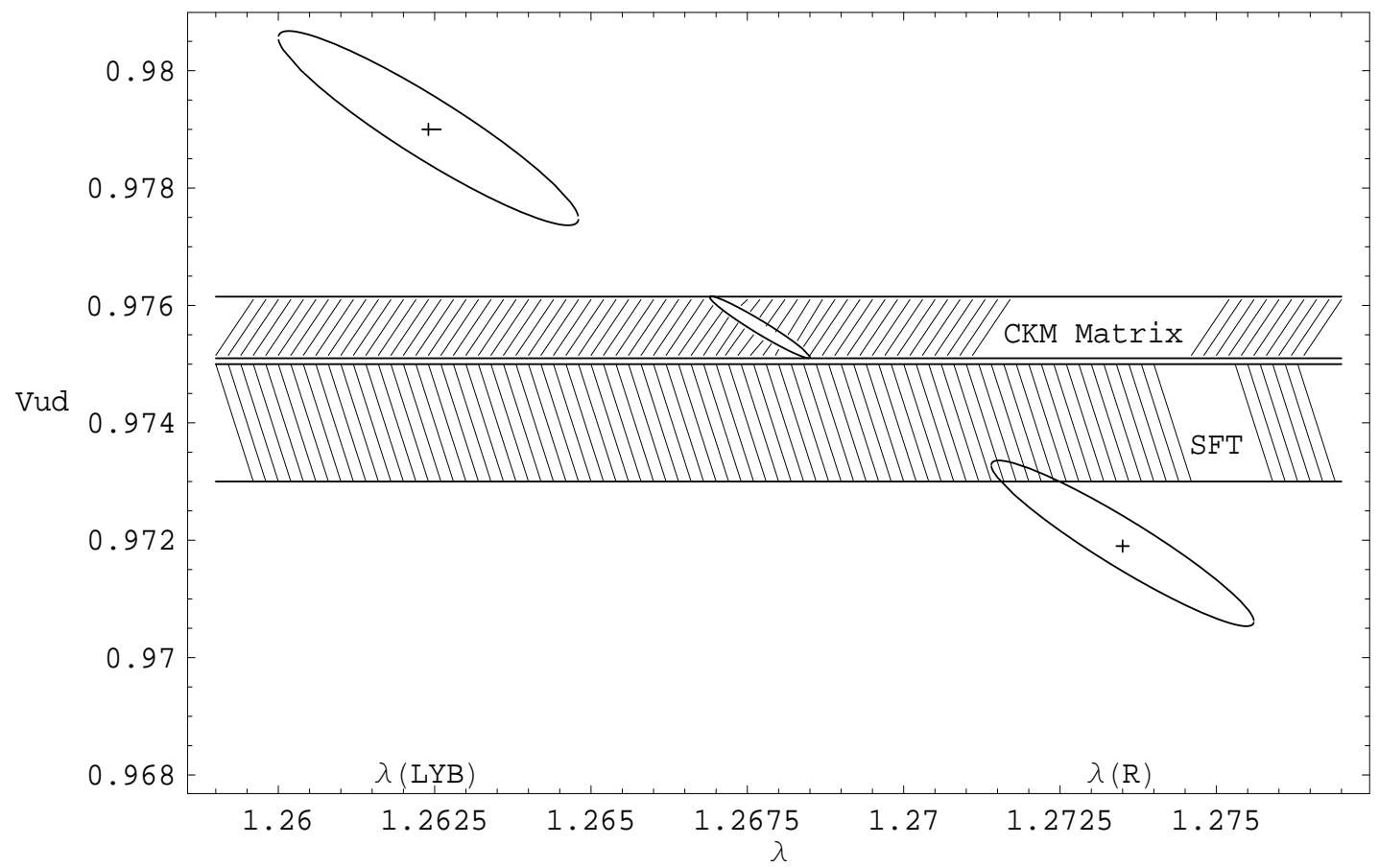

Fig. 1. $\left|V_{u d}\right|$ vs. $\lambda$. The two large ellipses corresponds to the $1 \sigma$ contours obtained via Eq. (20). We also display the two horizontal bands corresponding to $\left|V_{u d}\right|$ from unitarity of the CKM matrix and SFT at $1 \sigma$, too. The small ellipse within the CKM matrix band illustrates the potential of neutron beta decay to detect new physics or to put strong lower limits to its existence. 